\section{Methicillin-resistant Staphylococcus aureus}

\section{What is methicillin-resistant Staphylococcus aureus?}

Staphylococcus aureus (commonly known as 'staph') are common, usually harmless bacteria. Many healthy people carry these bacteria on their skin or in their nose. Sometimes, however, they can cause infection and serious illness. Some strains of staph are resistant to methicillin and other antibiotics. These are known as methicillinresistant Staphylococcus aureus (MRSA). MRSA infection is commonly known as 'golden staph'.

\section{What is community-acquired MRSA?}

MRSA infections occur frequently among people in hospitals and other health care facilities. Some MRSA strains, known as community-acquired MRSA (CaMRSA), spread readily between people in the community. CaMRSA strains are often quite different to MRSA strains associated with hospitals and may cause infections in otherwise healthy people.

\section{What are the symptoms?}

Like ordinary staph, CaMRSA can cause infections:

- of the skin surface (e.g. boils and impetigo (school sores))

- under the skin (e.g. abscesses and cellulitis)

- of the bone, blood, lungs and other parts of the body.

\section{How is it spread?}

CaMRSA can get into the body through broken skin or sores, resulting in redness, pimples, swelling, tenderness or boils. Infections can become serious, leading to infections of the blood or pneumonia. CaMRSA can be spread by:

- touching or squeezing an infected body area, such as a boil or open wound

- using towels, clothes or bed sheets that have been used by a person with an MRSA infection

- using grooming items that have been used by a person with an MRSA infection

- not washing your hands carefully.

\section{Who is at risk?}

CaMRSA skin infections can affect anyone. Crowding and frequent skin-to-skin contact can increase the risk of infection, so outbreaks tend to occur in schools, dormitories, military barracks, households, jails and child-care centres. Cuts or abrasions, contact with contaminated items and surfaces, and infrequent washing increase the risk of infection. People who have health problems such as diabetes or a poor immune system, or who have broken skin due to wounds or dermatitis, are also more likely to get an infection.

\section{How is it prevented?}

- Hand washing is important to prevent the spread of CaMRSA. Hands should be thoroughly washed with soap and running water for 10-15 seconds before and after touching or dressing an infected area, before handling or eating food, after going to the toilet, after blowing your nose and after touching or handling unwashed clothing or linen.

- Cover boils or other skin infections with a waterproof dressing. People who handle food must make sure that they do not contaminate any food and must keep any sores or skin infections completely covered with a waterproof dressing.

- Do not share personal items (e.g. clothes, towels or bed sheets) or grooming items (e.g. nail scissors, tweezers, razors and toothbrushes). If you share a bed with someone, keep sores or wounds covered overnight.

In addition to general hygiene, specific measures exist to help prevent the spread of MRSA in child-care centres, schools and among sporting groups.

\section{MRSA in child-care centres and schools}

- Teachers, children and families should understand the importance of hand washing, covering mouths while coughing and staying home if sick.

- Hand washing products (soap dispensers, running water and paper towel) should be available and accessible.

- Activities should allow time for hand washing (before eating and after going to the toilet).

- If open skin wounds cannot be kept covered, temporary exclusion from child care or school may be considered until the wound is healed or drainage of pus from the wounds can be contained using a sealed bandage.

- Surfaces such as counters, desks and toys that come into contact with uncovered or poorly covered infections should be cleaned daily with detergent, and whenever visibly contaminated.

\section{MRSA in sporting groups}

- People who have skin infections or open wounds that cannot be kept covered should not participate in contact sports until the wound has healed or drainage can be contained.

- People who have skin infections or open wounds should be excluded from common spas or saunas.

- People who have uncovered skin wounds should not share towels or sports equipment that is in contact with the skin. 
How is it diagnosed?

Staph infections are usually diagnosed on the basis of their appearance and the presence of any related symptoms (e.g. fever). To diagnose an infection of MRSA, a doctor will need to take a swab or sample from the boil, wound or other site of infection for laboratory testing.

\section{How is it treated?}

Your doctor will advise on the best treatment for your infection. Many CaMRSA skin infections can be treated by draining the abscess or boil. Letting the pus drain out safely is often the only treatment that is needed. Drainage of boils or abscesses should only be performed by a doctor, trained nurse or health worker under sterile conditions. It is important to keep the wound well protected with a waterproof bandage so the infection is not spread to others.

In some circumstances CaMRSA is treated with antibiotics. If you are given an antibiotic, take all doses as instructed, even if the infection improves. It is possible for a CaMRSA skin infection to come back after it appears cured.

\section{What is the public health response?}

Public health units can advise on the control of outbreaks. CaMRSA is not a notifiable condition in NSW.

For more information please contact your doctor, local public health unit or community health centre.

This factsheet is available at: http://www.health.nsw.gov.au/ factsheets/infectious/methicilresist_staph.html 